\title{
论 文
}

\section{磁场影响聚合物本体结晶动力学的机理分析}

\author{
刘丽 ${ }^{(1) * *}$ ，吴文潭 ${ }^{1}$ \\ (1) 上海大学材料学院高分子材料系, 上海 201800 \\ (2) 中空纤维膜材料与膜过程教育部重点实验室; 天津工业大学 天津 300160 \\ *通讯作者, E-mail: liuli@staff.shu.edu.cn
}

收稿日期: 2011-03-09; 接受日期: 2011-04-26; 网络版发布日期: 2011-07-23 doi: 10.1360/032011-139

\begin{abstract}
摘要 在经典的热力学理论基础上, 探讨了磁场对聚合物本体结晶过程的成核与生长的影 响, 建立了相关结晶动力学理论方程. 初步认为, 磁场产生的“磁结晶效应”可能是由于晶相 与非晶相之间磁化率差异导致了两相之间磁化能的差异, 也可能由于聚合物体系在结晶前会 形成一种有序相, 减小了体系的熵值, 进而改变了结晶过程中的体系自由能, 影响其成核与 晶体生长, 乃至整个结晶动力学方程. 利用 Matlab 软件结合 PLLA 的各结晶参数值, 绘制了 结晶自由能与各成核临界参数之间的函数图像. 结果表明, 在低过冷度下, 较小的自由能扰 动可能导致较大的晶核临界参数变化.
\end{abstract}

关键词

磁场

聚合物本体结晶

热力学

Matlab 软件

\section{1 引言}

磁场对小分子溶液结晶的研究已较为成熟 ${ }^{[1]}$. 研 究中发现, 静磁场可以影响小分子结晶动力学 ${ }^{[2,3]}$, 改变小分子结晶的成核率与晶体尺寸, 如, 静磁场可 影响 $\mathrm{CaCO}_{3}$ 的溶液结晶, 导致 $\mathrm{CaCO}_{3}$ 晶粒数目变少, 晶粒尺寸变大 ${ }^{[4]}$. 这些研究对于小分子物质结晶工艺 的控制有着重要的意义.

磁场对聚合物结晶过程的影响研究较少, 近年, 已引起科学家的高度关注. 众所周知, 聚合物大都为 抗磁性物质, 磁化率较小. 传统的观点认为, 外磁场 对于聚合物本体结晶过程一般不能产生可观测到的 影响. 随着国内外学者研究工作的开展, 发现在磁场 的作用下, 聚合物的聚集态结构会发生微妙的变化. Kimura 和 Thomas 等人 ${ }^{[5-9]}$ 发现强磁场可以诱使聚合

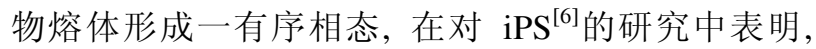
磁场可以加快 iPS 的结晶速率. 刘强 ${ }^{[10]}{ }^{10}$ 研究了静磁场 下等规聚丙烯薄膜熔体结晶的显微结构发现, 静磁 场的影响导致了聚丙烯球晶尺寸增加, 球晶密度降
低, 说明磁场改变了聚丙烯的结晶成核与生长速率. 滕莹雪等人 ${ }^{[11]}$ 通过观察强磁场下聚甲醛结晶后晶体 的显微结构, 发现聚甲醛片晶厚度有所增加, 其晶片 的生长过程受到了强磁场的影响. 这些研究结果表 明, 静磁场可以直接影响聚合物结晶的成核率与晶 体生长速率, 进而影响其结晶动力学. 俄罗斯科学家 Levin 等人曾在 2001 2003 年报道过关于高频脉冲磁 场对 SKTV、PDMS、PEO 三种聚合物结晶行为的影 响的研究工作 ${ }^{[12 \sim 14]}$. 他的研究有着相似的实验现象, 即聚合物经脉冲磁场的处理后再结晶, 晶体的成核 点增多, 晶体的尺寸减小, 出现了晶体细化, 他将磁 场这些作用统称为 “磁结晶效应”. 纵观近年来在这 一领域的研究成果可以发现, 一些聚合物在磁场中 晶体尺寸和成核点密度都发生了变化. 聚合物在静 磁场与脉冲磁场中表现的结晶过程的改变很有可能 具有相似的机理.

目前, 有关磁场对聚合物结晶的影响机理鲜见 报道, 尚处于探索阶段. 本文在经典热力学和现有其 他外场下聚合物结晶理论的基础上 ${ }^{[15]}$, 尝试建立了 
磁场下, 聚合物结晶过程中聚合物晶体成核与生长 速率的理论, 将“磁结晶效应”这一概念扩展到整个 磁场领域, 即指的是磁场对聚合物结晶过程施加影 响, 导致聚合物晶体尺寸与成核点密度发生变化的 一种效应. 并对“磁结晶效应”进行了初步解释. 应用 Matlab 软件, 进行聚合物 PLLA 结晶过程中的成核临 界参数 (自由能、链节数与链节长度)的三维函数计算 机图像绘制, 直观反映了各个成核临界参数与熔融 体自由能间关系, 推测磁场对聚合物结晶过程的影 响与热处理条件可能具有一种依赖关系.

\section{2 磁场对聚合物一次成核率的影响}

聚合物一次成核率直接与临界成核自由能有关. 基于经典的相变热力学方程, 及磁场下成核自由能 的变化来源于体系获得的磁化能, 初步探讨磁场对 聚合物一次成核率的影响规律.

\section{1 磁场下的相变热力学}

\subsection{1 经典相变热力学方程}

根据经典热力学, 当一个封闭体系中温度和压 力为定值时, 物质的相变自由能可写为:

$$
F=H-T S
$$

$H$ 为焓, $S$ 为熵, $T$ 为温度.

当整个系统发生相转变过程时, 即聚合物开始 结晶时, 体系的自由能将发生变化, 其中, 非晶相, 即无定形相的自由能为:

$$
F_{\mathrm{a}}=H_{\mathrm{a}}-T S_{\mathrm{a}}
$$

晶相的自由能为:

$$
F_{\mathrm{c}}=H_{\mathrm{c}}-T S_{\mathrm{c}}
$$

由(3)式减(2)式之差可得相变自由能差为:

$$
\Delta F=\Delta H-T \Delta S
$$

\subsection{2 磁场下体系相变热力学方程}

当施加一个外磁场时, 两相之间将获得不同的 磁化能，其微分式可表达如下:

$$
\begin{aligned}
& \mathrm{d} F_{\mathrm{a}}=-\frac{1}{\mu_{0}} V M_{\mathrm{a}} \mathrm{d} B \\
& \mathrm{~d} F_{\mathrm{c}}=-\frac{1}{\mu_{0}} V M_{\mathrm{c}} \mathrm{d} B
\end{aligned}
$$

其中, $\mathrm{d} F_{\mathrm{a}}$ 为非晶相获得的磁化能的微分, $\mathrm{d} F_{\mathrm{c}}$ 为晶相
获得的磁化能的微分, $B$ 为磁感强度, $M_{\mathrm{a}}$ 与 $M_{\mathrm{c}}$ 分别 为聚合物非晶相与晶相的单位体积磁化强度, $V$ 是 体积, $\mu_{0}=4 \pi \times 10^{-7}(\mathrm{H} / \mathrm{m})$ 为真空磁导率, 式中磁 化强度定义为:

$$
M=x B
$$

其中, $x$ 为磁化率.

将式(5)和(6)积分便可得聚合物两相在磁场中各 自获得的磁化能为:

$$
\begin{aligned}
& \Delta F_{\mathrm{a}}=-\frac{1}{2 \mu_{0}} V x_{\mathrm{a}} B^{2} \\
& \Delta F_{\mathrm{c}}=-\frac{1}{2 \mu_{0}} V x_{\mathrm{c}} B^{2}
\end{aligned}
$$

式中, $\Delta F_{\mathrm{a}}$ 为非晶相获得的磁化能, $\Delta F_{\mathrm{c}}$ 为晶相获得 的磁化能; $x_{\mathrm{a}}$ 与 $x_{\mathrm{c}}$ 分别为非晶相与晶相的单位体积 抗磁磁化率.

式(8)(9)之差即为磁场对于两相之间自由能差的 贡献:

$$
\begin{aligned}
\Delta F_{\mathrm{m}} & =\Delta F_{\mathrm{c}}-\Delta F_{\mathrm{a}}=-\frac{1}{2 \mu_{0}} V\left(x_{\mathrm{c}}-x_{\mathrm{a}}\right) B^{2} \\
& =-\frac{1}{2 \mu_{0}} V \Delta x B^{2}
\end{aligned}
$$

其中 $\Delta x$ 即为两相间的单位体积抗磁磁化率差.

结合式(4)与(10), 可写出磁场下体系相变热力 学方程:

$$
\Delta F^{\prime}=\Delta H-T \Delta S-\frac{1}{2 \mu_{0}} V \Delta x B^{2}
$$

\section{2 成核理论}

\subsection{1 传统的聚合物成核热力学理论}

传统的均相成核理论 ${ }^{[16]}$ 表明: 结晶过程中, 晶 相生长是晶体的体自由能与表面自由能竞争的过程, 其中体自由能是结晶的动力, 而晶体的表面自由能 则阻碍晶体的生长, 结晶的总自由能变化 $\Delta F$ 可由下 式表示:

$$
\Delta F=-V \Delta f+A \sigma
$$

其中, $V$ 为体系中的晶体体积, $\Delta f$ 为单位体积晶体 的熔融自由能, $A$ 为体系中两相界面的总面积, $\sigma$ 为单位表面积的相界面自由能.

对于聚合物而言，结晶初期，晶体都是捆束状 的 $^{[17]}$, 因而可以将聚合物晶核视为许多高分子链节 


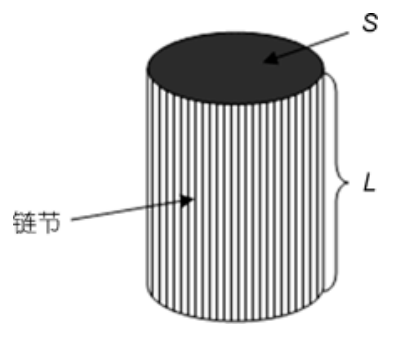

图 1 圆柱状晶核示意图

捆束的圆柱体处理, 如图 1 所示. 对于形成由 $N$ 个链 节组成的柱状临界晶核(设每个链节的长度均为 $L$, 上表面面积为 $S$, 定义 $A=S / N$, 即上下表面单位链 平均所占有的面积)形成自由能变化 $\Delta F_{0}$ 为:

$$
\Delta F_{0}=F_{\mathrm{c}}-F_{\mathrm{a}}=-N L A \Delta f+2 N A \sigma_{e}+2 L \sqrt{N A \pi} \sigma
$$

式中, $\sigma_{\mathrm{e}}$ 为晶核的末端单位面积表面自由能, $\sigma$ 为 晶核侧表面单位面积自由能, $F_{\mathrm{c}}$ 为晶相的自由能, $F_{\mathrm{a}}$ 为非晶相的自由能.

可由 $\frac{\partial \Delta F}{\partial L}=0$, 得到临界折叠链节数 $N^{*}$ :

$$
N^{*}=\frac{4 \pi \sigma^{2}}{A \Delta f^{2}}
$$

由 $\frac{\partial \Delta F}{\partial N}=0$ 再结合式(14), 可得到临界晶核长度 $L^{*}$ :

$$
L^{*}=\frac{4 \sigma_{\mathrm{e}}}{\Delta f}
$$

将(14)与(15)式代入(13)式, 进而可得到临界晶 核自由能 $\Delta F^{*}$ :

$$
\Delta F^{*}=\frac{8 \pi \sigma_{\mathrm{e}} \sigma^{2}}{\Delta f^{2}}
$$

\subsection{2 磁场中聚合物成核热力学理论}

在半结晶体系中, 设 $x_{\mathrm{c}}$ 与 $x_{\mathrm{a}}$ 分别为晶相与非晶 相聚合物的磁化率. $x$ 为整个体系的等效复合磁化 率, 由于聚合物是弱磁质, 可近似认为体积为 $V$ 的聚 合物中, 晶区 (体积为 $V_{\mathrm{c}}$ ) 和非晶区 (体积为 $V_{\mathrm{a}}$ ) 的磁感 应强度与总磁感应强度一致都为 $B$ (忽略相互的磁屏 蔽).

则三个磁化率的关系可由下两式给出:

$$
\begin{gathered}
\frac{V x B^{2}}{2 \mu_{0}}=\frac{1}{2 \mu_{0}}\left(V_{\mathrm{c}} x_{\mathrm{c}} B^{2}+V_{\mathrm{a}} x_{\mathrm{a}} B^{2}\right) \\
V=V_{\mathrm{c}}+V_{\mathrm{a}}
\end{gathered}
$$

结合(17)、(18)两式, 得到:

$$
V x=V_{\mathrm{c}} x_{\mathrm{c}}+V_{\mathrm{a}} x_{\mathrm{a}}
$$

可转化为:

$$
\begin{aligned}
x & =f_{\mathrm{V}} x_{\mathrm{c}}+\left(1-f_{\mathrm{V}}\right) x_{\mathrm{a}} \\
& =f_{\mathrm{V}}\left(x_{\mathrm{c}}-x_{\mathrm{a}}\right)+x
\end{aligned}
$$

式中,

$$
f_{\mathrm{V}}=\frac{n N A L}{V}
$$

$f_{\mathrm{V}}$ 为晶区所占的体积分数, $n$ 为晶核数.

则一个半结晶体与等体积的非晶体的自由能差 $\Delta F_{\mathrm{v}}$ 由下式给出:

$\Delta F_{\mathrm{v}}=V\left(F-F_{\mathrm{a}}\right)$

$=V\left[\left(F-\frac{1}{2 \mu_{0}} x B^{2}\right)-\left(F_{\mathrm{a}}-\frac{1}{2 \mu_{0}} x_{\mathrm{a}} B^{2}\right)\right]$

$=V f_{\mathrm{v}}\left[\left(F_{\mathrm{c}}-F_{\mathrm{a}}\right)-\frac{1}{2 \mu_{0}}\left(x_{\mathrm{c}}-x_{\mathrm{a}}\right) B^{2}\right]$

$=n\left[-N L A \Delta f+2 N A \sigma_{\mathrm{e}}+2 L \sqrt{N A \pi} \sigma-\frac{N A L}{2 \mu_{0}}\left(x_{\mathrm{c}}-x_{\mathrm{a}}\right) B^{2}\right]$

式(22)中, $F$ 为半结晶体系的总自由能.

由上式可得单核体系, 即 $n=1$ 时的自由能变化 为:

$$
\begin{aligned}
\Delta F= & -N L A \Delta f+2 N A \sigma_{\mathrm{e}}+2 L \sqrt{N A \pi} \sigma \\
& -\frac{N A L}{2 \mu_{0}}\left(x_{\mathrm{c}}-x_{\mathrm{a}}\right) B^{2}
\end{aligned}
$$

由此可得:

$$
\begin{aligned}
\Delta F= & -N L A\left(\Delta f+\frac{1}{2 \mu_{0}}\left(x_{\mathrm{c}}-x_{\mathrm{a}}\right) B^{2}\right)+2 N A \sigma_{\mathrm{e}} \\
& +2 L \sqrt{N A \pi} \sigma
\end{aligned}
$$

即

$$
\Delta F=-N L A\left(\Delta f-\Delta F_{\mathrm{m}}\right)+2 N A \sigma_{\mathrm{e}}+2 L \sqrt{N A \pi} \sigma
$$

上式说明, 磁场的存在对体系引入了磁化能进而改 变了熔融体自由能.

根据式(25)可得到磁场下聚合物的成核临界参 
数如下:

$$
\begin{aligned}
N_{\mathrm{m}}{ }^{*} & =\frac{4 \pi \sigma^{2}}{A\left(\Delta f-\Delta F_{\mathrm{m}}\right)^{2}} \\
L_{\mathrm{m}}{ }^{*} & =\frac{4 \sigma_{\mathrm{e}}}{\Delta f-\Delta F_{\mathrm{m}}} \\
\Delta F_{\mathrm{m}}{ }^{*} & =\frac{8 \pi \sigma_{\mathrm{e}} \sigma^{2}}{\left(\Delta f-\Delta F_{\mathrm{m}}\right)^{2}}
\end{aligned}
$$

在低过冷度的情况下, $\Delta f$ 可由下式得到:

$$
\begin{aligned}
\Delta f & =\Delta h-T_{\mathrm{m}} \Delta S \quad \approx \Delta h\left(1-T_{\mathrm{m}} / T_{\mathrm{m}}{ }^{0}\right) \\
& =\Delta h \Delta T / T_{\mathrm{m}}{ }^{0}
\end{aligned}
$$

式中, $T_{\mathrm{m}}{ }^{0}$ 为平衡熔化温度, $\Delta h$ 为熔融焓值, $\Delta T$ 为 过冷度, $\Delta T=T_{\mathrm{m}}{ }^{0}-T$.

以上分析可知, 体系在相变时, 可以从磁场中获 得一定的磁化能差作为相变的一个 “额外驱动力”, 使成核过程发生一定的变化. 尽管聚合物是弱磁性 物质, 这一磁化能可能相对较小, 但是在过冷度较小 的情况下, 即系统原体积自由能较小的情况下, 磁化 能的作用可能会表现出来.

\subsection{Matlab 软件分析成核自由能与各成核临界参 数的关系}

由 2.2.2 部分的推导可见, 成核自由能的改变将 直接影响各成核临界参数值, 见式(26) (28), 表明磁 场的参与, 改变了聚合物结晶过程中两相间的熔融 体自由能项, 从而改变了成核的自由能. 为了更直观 地了解成核过程中成核自由能与各临界参数的关系, 本文以 PLLA 为例, 依据 PLLA 的相关参数 (表 1), 用 Matlab 描绘出低过冷度下成核自由能与各临界参数 之间的关系的函数图像.

PLLA 的晶格参数为 $a=1.07 \mathrm{~nm}, b=0.61 \mathrm{~nm}^{[18]}$, 为简化计算, 假设 $A=1 \mathrm{~nm}^{2}$, 单位体积熔融焓 $\Delta h$ 是

表 1 PLLA 的一些相关参数 ${ }^{[18,19]}$

\begin{tabular}{cc}
\hline 参数 $($ 单位 $)$ & 参数值 \\
\hline$\sigma_{e}\left(\mathrm{~J} \mathrm{~m}^{-3}\right)$ & $61.2 \times 10^{-3}$ \\
$\sigma\left(J \mathrm{~m}^{-3}\right)$ & $12.0 \times 10^{-3}$ \\
$T_{m}{ }^{0}\left({ }^{\circ} \mathrm{C}\right)$ & 480 \\
$\Delta T\left({ }^{\circ} \mathrm{C}\right)$ & 20 \\
$\Delta h\left(J \mathrm{~m}^{-3}\right)$ & 120.74 \\
$\Delta h_{m}\left(\mathrm{~J} \mathrm{~g}^{-1}\right)$ & 93.60 \\
\hline
\end{tabular}

通过单位质量熔融焓 $\Delta h_{\mathrm{m}}$ 与 PLLA 密度 $\rho_{\text {PLLA }}$ 的乘积 得到. 设晶核的链节数 $N$ 为连续值, 代入(13)式, 得 到 PLLA 总结晶自由能的一个函数式, 用 Matlab 软件 描绘出函数图像, 函数的 $X, Y$ 轴分别为链节数 $N$ 和 链节长度 $L$, 如图 2 所示:

根据图 2 可以看出, 随着 $L$ 与 $N$ 数值的增加, 自 由能变化趋向于负值, 即更有利于晶体成核, 当 $L$ 与 $N$ 增大到一定数值时, 越过曲面的临界平面(此面法线与 $\mathrm{Z}$ 轴平行), 晶核趋于稳定. 图 2 的三视图见图 3 .

图 3 中各图的红圈所标即对应着此状态下(过冷 度 $\Delta T=20{ }^{\circ} \mathrm{C}$ ) 的临界成核点位置, 根据图 3(c) 可见, $L$ 的数值约为 $51 \mathrm{~nm}, N$ 约为 76 个. 此结果与将表 1 数值代入式(26)(27)的计算结果吻合.

根据式(29)可知, 改变过冷度的数值可以改变晶 体的熔融体积自由能, 设结晶的过冷度分别为 20,24 和 $28{ }^{\circ} \mathrm{C}$, 用 Matlab 做出相应的函数图像, 如图 4 所 示:

图 4 为自由能曲面随体自由能变化的示意图, 其 中(a)、(b)、(c)分别为其全视图、 $X Z$ 面视图和 $Y Z$ 面 视图. 由图 4 可见, 在过冷度较小时, 如 $20{ }^{\circ} \mathrm{C}$ 到 $28{ }^{\circ} \mathrm{C}$, 只有 $8{ }^{\circ} \mathrm{C}$ 较小的过冷度变化, 也可能对各临 界参数产生较大的影响, 如链段数 $N$ 由 76 逐渐变化 到 39, 减小了将近一半. 可以预见, 磁场的存在相对 于体自由能而言产生了一个较小的磁化能, 在低过 冷度的情况下, 也可能对成核临界参数造成较大的 影响, 进而影响成核过程.

\section{4 “似液晶相”及对成核自由能的影响}

磁场可能在聚合物结晶的诱导期, 诱导其形成

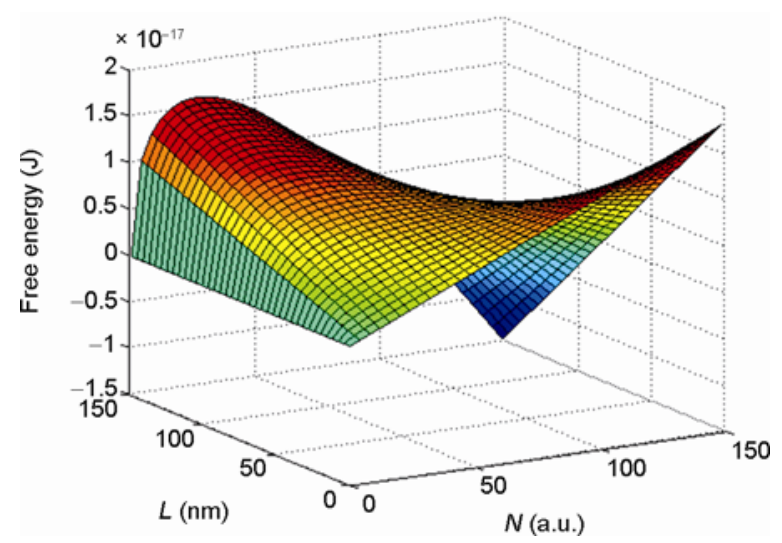

图 2 成核过程中总结晶自由能变化的函数图像. $X, Y$ 轴分 别为链节数 $N$ 和链节长度 $L . Z$ 轴为结晶自由能 


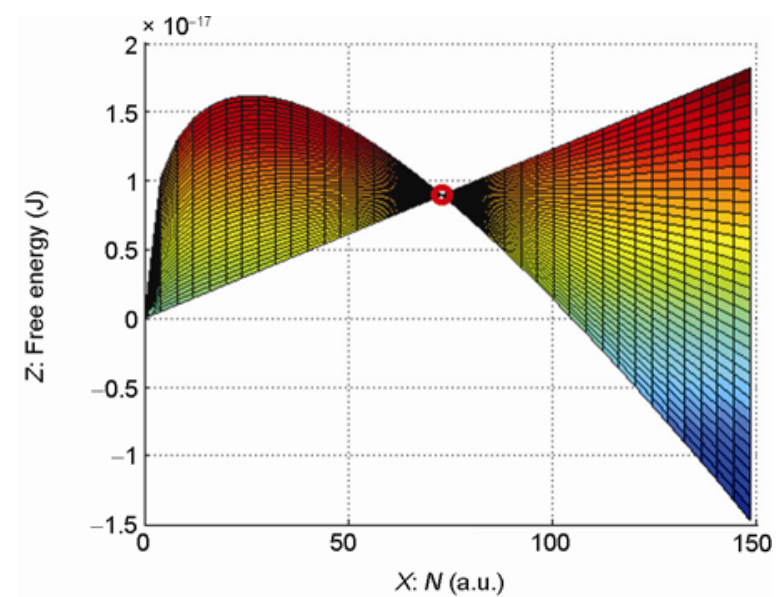

(a) $X Z$ 面

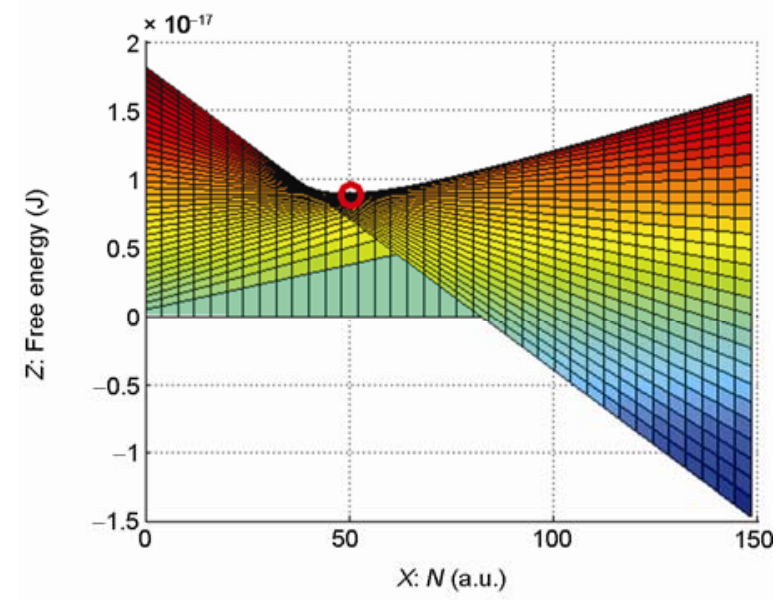

(b) $Y Z$ 面

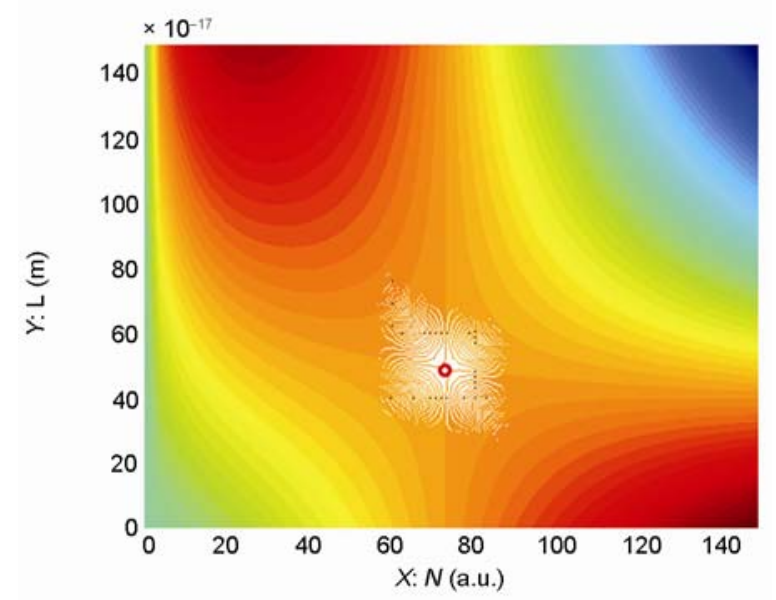

(c) $X Y$ 面 等高线视图

图 3 总自由能函数图像(图 2)的三视图, (a)(b)(c) 分别是图 2 的 $X Z 、 Y Z 、 X Y$ 面的视图, 其中 (c)为等高线视图

一个有序结构的相态. 日本学者 Kimura 研究发现, 磁场诱导 $\mathrm{PEN}^{[5]} 、 \mathrm{iPS}^{[6]} 、 \mathrm{iPP}^{[7]} 、 \mathrm{PET}^{[8]}$ 形成了一种较

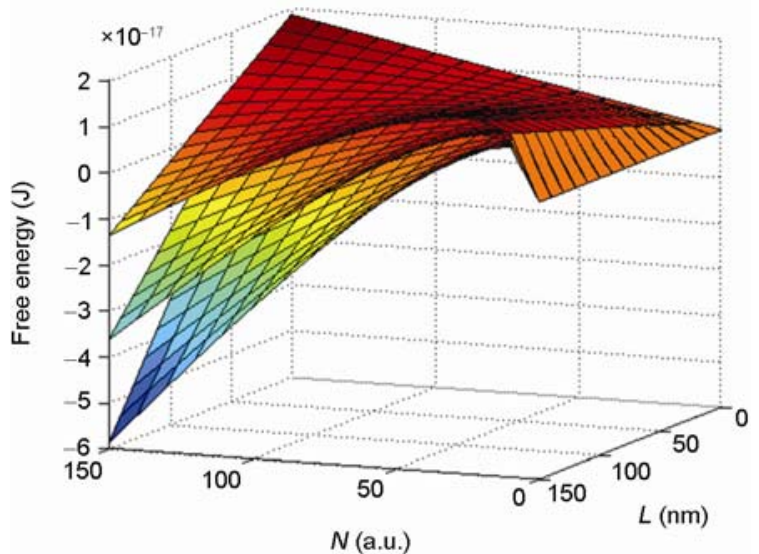

(a) 全视图

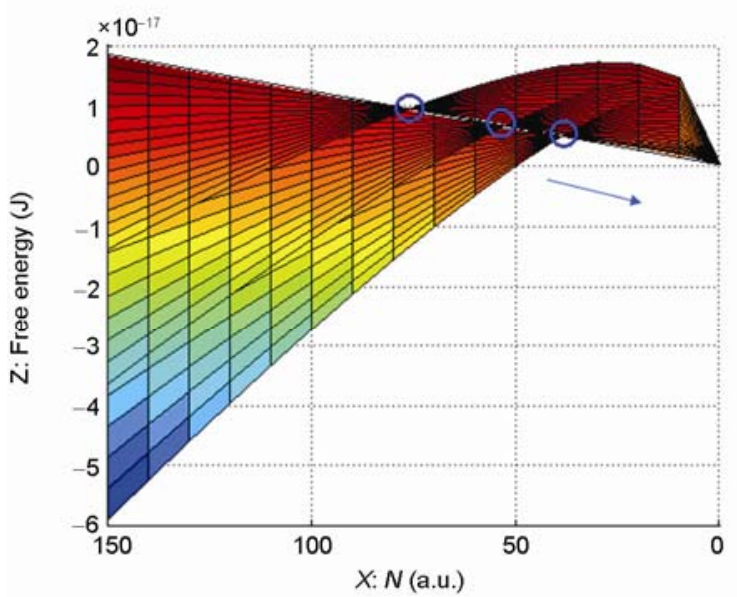

(b) $X Z$ 面视图

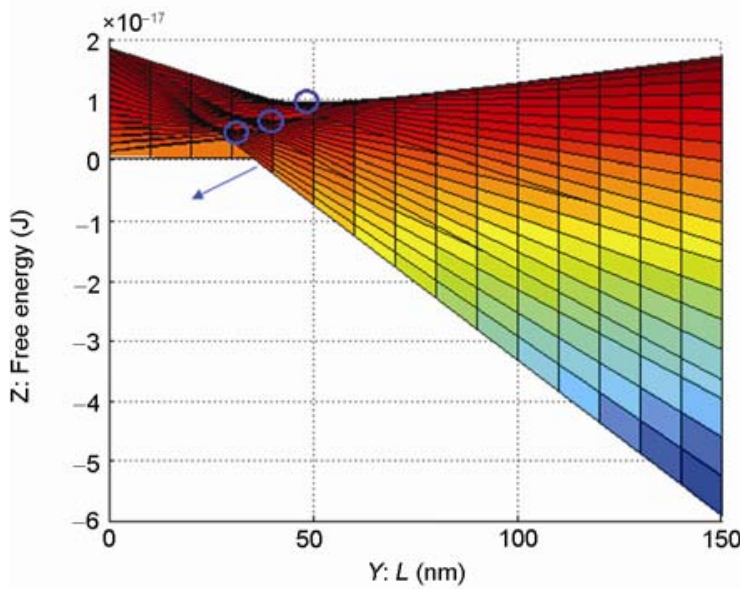

(c) $Y Z$ 面视图

图 4 自由能曲面随体自由能变化的图示. (a)全视图; (b) $X Z$ 面视图; (c) $Y Z$ 面视图. 三幅图像, 由上到下的过冷度, 都分 别为 20,24 和 $28{ }^{\circ} \mathrm{C}$. 篮圈标注的是各临界成核点位置, 箭 头指向的是随着过冷度的增大临界点的变化方向

无定形态更为有序的相态，称其为“似液晶相”. 磁场 
对聚合物的这一效应可能是由于这些聚合物晶体本 身具有各向异性的磁化率. 研究者就其形成进行了 揭示, 这种有序相结构是在晶粒的熔融过程中形成 的, 随着晶粒的尺寸逐渐减小, 当尺寸在一个合适的 范围内, 晶粒可以克服黏阻力与热扰动, 其中磁化率 较小的方向可以沿磁场取向. 即使晶粒完全熔融, 在 一段时间内, 一部分熔体链段仍会保持取向排列, 形 成了“似液晶相”。研究发现 ${ }^{[7]}$, 在磁场下, iPP 的成核 初期, 最先形成的晶核基本朝向磁场方向, 这可能是 由于取向部分的有序结构——“似液晶相”优先成核, 在一定程度上预示这种有序结构将改变体系的结晶 过程.

当磁场诱导聚合物形成了“似液晶相”后, 聚合 物的常规相变, 即非晶相与结晶两相相变过程就转 变为 “似液晶相”与晶体相两相的相变过程. 其相变 过程的热力学分析如下:

非晶相的单位体积自由能为:

$$
F_{\mathrm{a}}=H_{\mathrm{a}}-T S_{\mathrm{a}}
$$

“似液晶相”的单位体积自由能为:

$$
F_{\mathrm{a}^{\prime}}=H_{\mathrm{a}^{\prime}}-T S_{\mathrm{a}^{\prime}}
$$

晶相的单位体积自由能为:

$$
F_{\mathrm{c}}=H_{\mathrm{c}}-T S_{\mathrm{c}}
$$

且有关系式: $H_{\mathrm{a}}>H_{\mathrm{a}^{\prime}}>H_{\mathrm{c}}$ 和 $S_{\mathrm{a}}>S_{\mathrm{a}^{\prime}}>S_{\mathrm{c}}$,

其中, $H_{\mathrm{a}} 、 H_{\mathrm{a}^{\prime}} 、 H_{\mathrm{c}}$ 是各相的单位体积焓, $S_{\mathrm{a}} 、 S_{\mathrm{a}^{\prime}}$ 、 $S_{\mathrm{c}}$ 是各相的单位体积熵.

结合(30)、(31)、(32)式可得晶相与无定形相之间 单位体积熔融自由能为:

$$
\Delta f=F_{\mathrm{a}}-F_{\mathrm{c}}=\left(H_{\mathrm{a}}-H_{\mathrm{c}}\right)-T\left(S_{\mathrm{a}}-S_{\mathrm{c}}\right)
$$

晶相与 “似液晶相”之间单位体积熔融自由能 $\Delta f^{\prime}$ 为:

$$
\Delta f^{\prime}=F_{\mathrm{a}^{\prime}}-F_{\mathrm{c}}=\left(H_{\mathrm{a}^{\prime}}-H_{\mathrm{c}}\right)-T\left(S_{\mathrm{a}^{\prime}}-S_{\mathrm{c}}\right)
$$

由式(33)和(34)可得:

$$
\Delta f^{\prime}-\Delta f=\left(H_{\mathrm{a}^{\prime}}-H_{\mathrm{a}}\right)-T\left(S_{\mathrm{a}^{\prime}}-S_{\mathrm{a}}\right)
$$

由式(35)可见，晶相与“似液晶相”之间单位体积 熔融自由能, 和晶相与非晶相之间的单位体积熔融 体自由能有所变化, 当 $T$ 为常数时, 这一变化是由非 晶相与 “似液晶相”两相焓变与两相熵变共同决定的. 聚合物一般都呈现软物质特性 ${ }^{[20]}$ ，当其受到外作用 时, 体系熵变常常远大于焓变, 所以本文采取近似处 理的方法, 将焓变项忽略, 认为似液晶相主要是熵致
相变, 由式(35)可得下式:

$$
\Delta f^{\prime}=\Delta f-T\left(S_{\mathrm{a}^{\prime}}-S_{\mathrm{a}}\right)
$$

因为 $S_{\mathrm{a}}>S_{\mathrm{a}^{\prime}}$, 所以聚合物相变的熔融自由能 $\Delta f^{\prime}$ 增加了, 即结晶自由能减小, 结晶变得容易. 由 此可见，由于磁场诱导有序相的作用有利于体系结 晶.

\section{5 磁场下的一次成核速率方程}

根据经典的聚合物成核速率理论，聚合物的晶 体的成核速率方程简单表达如下:

$$
I_{0}=k^{\prime} \exp \left[\frac{-U^{*}}{k\left(T-T_{\infty}\right)}\right] \exp \left(-\frac{\Delta F^{*}}{k T}\right)
$$

式中 $k^{\prime}$ 为常数, $U^{*}$ 为链段传输所需的激活能, $\Delta F^{*}$ 为临界成核自由能, $k$ 为波尔兹曼常数, $T$ 为结 晶温度, $T_{\infty}=T_{g}-k_{3}, T_{g}$ 为玻璃化转变温度, $k_{3}$ 为常 数, 其数值一般为 30 .

当施加磁场时, 由 2.2 部分可知, 磁场诱导体系 的结晶自由能发生了变化. 如果体系的结晶方式是 本体结晶, 而聚合物为绝缘体, 因而可假设晶体生长 中链段传输受磁场的影响可忽略不计, 即磁场不改 变传输能. 此时, 其成核率由 $I_{0}$ 变为 $I_{m}$, 成核临界自 由能即由 $\Delta F^{*}$ 变为 $\Delta F_{m}{ }^{*}$, 则有磁场下成核速率方程:

$$
I_{m}=k^{\prime} \exp \left[\frac{-U^{*}}{k\left(T-T_{\infty}\right)}\right] \exp \left(-\frac{\Delta F_{m}{ }^{*}}{k T}\right)
$$

结合式(37)、(38)和(28)可得:

$$
\ln \left(\frac{I_{m}}{I_{0}}\right)=\frac{8 \pi \sigma_{e} \sigma^{2}}{k T}\left[\left(\frac{1}{\Delta f}\right)^{2}-\left(\frac{1}{\Delta f-\Delta F_{m}}\right)^{2}\right]
$$

结合式(29)和(39)可得:

$$
\ln \left(\frac{I_{m}}{I_{0}}\right)=\frac{8 \pi \sigma_{e} \sigma^{2} T_{m}^{02}}{k T \Delta T^{2}}\left[\left(\frac{1}{\Delta h}\right)^{2}-\left(\frac{1}{\left.\left.\Delta h-\frac{T_{m}^{0} \Delta F_{m}}{\Delta T}\right)^{2}\right]}\right.\right.
$$

由上述分析，再结合式(10)可得如下结果，在一 定过冷度 $\Delta T$ 下, 当 $x_{c}>x_{a}$ 时, 随着磁感强度 $B$ 增加, $\Delta F_{m}$ 变小, $\ln \left(I_{m} / I_{0}\right)$ 数值变大, 成核率增加, 即 $I_{m}>I_{0}$; 当 $x_{c}<x_{a}$ 时, 随着磁感强度 $B$ 增加 $\Delta F_{m}$ 变 大, $\ln \left(I_{m} / I_{0}\right)$ 数值变小, 成核率降低, 即 $I_{\mathrm{m}}<I_{0}$. 
当磁场诱导聚合物形成“似液晶相”结构, 则有:

$\ln \left(\frac{I_{m}}{I_{0}}\right)=\frac{8 \pi \sigma_{\mathrm{e}} \sigma^{2}}{k T}\left[\left(\frac{1}{\Delta f}\right)^{2}-\left(\frac{1}{\Delta f-T\left(S_{a^{\prime}}-S_{a}\right)-\Delta F_{m}}\right)^{2}\right]$

由式(41)可见, 似液晶相的存在, 导致熔融体自 由能增大, 体系的成核率增加.

综上所述，对于同一聚合物而言，如果两相间磁 化率差值较大, 或者磁场诱导似液晶相的形成, 导致 体系熵值变化较大, 都会影响成核率. 其中 $x_{c}<x_{a}$ 时, $\Delta F_{m}>0$, 由两相磁化率差和“似液晶相”结构产 生的效应互相削弱, 成核率宏观上可能不表现出明 显改变, 但是当这两种效应的叠加对于相变自由能 的改变十分显著时, 磁场对于成核速率的影响便不 能忽略, 可以对成核速率产生较大变化. 当 $x_{c}>x_{a}$ 时, 或者, 磁场诱导聚合物产生似液晶相结构, 那么 随着磁场强度 $B$ 的增加, 成核率总是增加的, 如果这 两种效应叠加后作用十分明显, 那么成核速率便会 产生更大变化. 需要指出的是, “似液晶相”在一定的 条件下才能形成 ${ }^{[9]}$, 且两相之间的磁化能可能非常 小, 必须在过冷度较小时才能表现出成核速率的可 观测到的改变, 这说明, 磁场所产生的这种成核速率 变化对结晶温度有一定的依赖性.

\section{3 磁场下聚合物晶体的生长.}

前面尝试建立了磁场影响聚合物晶体一次成核 的理论. 理论表明, 磁场的存在在一定条件下, 可能 导致成核速率的明显改变. 聚合物的结晶过程除了 一次成核外还有晶体生长的过程. 磁场的作用, 也会 不同程度地影响到晶体的生长.

经典晶体生长过程理论为 Lauritzen-Hoffman 理 论(简称 L-H 理论). 其理论方程是用以解释线型的柔 性大分子熔体结晶 (由无定形体转变为折叠链晶片) 的结晶动力学最为普遍的方程之一 ${ }^{[21]}$. 这个理论以 及它的许多修正理论在聚合物多分子结晶行为的解 释与建模方面, 构成了最全面而广泛的方法体系. 它 从第一次提出至今, 已经被大大地改进和发展, 吸收 了更多的新概念以扩大它的使用领域, 满足在这些 领域的其他研究. 它不仅可以很好地符合以及描述 PE 和其他柔性聚合物的折叠链结晶过程, 而且, 也 较成功地应用于一些刚性链体系, 如 PEEK. 理论的
推导方法是用阶梯式的方式处理片晶的生长, 即从 考虑第一个链节的沉积位置开始, 然后处理随后的 链节沉积. 由于 L-H 理论的具体推导十分复杂 ${ }^{[22]}$, 下 面仅给出最终方程形式:

$$
\begin{gathered}
G=G_{i} \exp \left(-U^{*} / R\left(T_{c}-T_{\infty}\right)\right) \exp \left(-\frac{j \sigma \sigma_{e}}{K T_{c} \Delta f}\right) \\
G_{i}=\frac{N_{0} b_{0} J}{l_{u}}\left[\frac{k T_{c}}{b_{0} \sigma}-\frac{k T}{2 b_{0} \sigma+a_{0} b_{0} \Delta f}\right]
\end{gathered}
$$

式(42)中, $G$ 为聚合物晶片的末端生长速度 (通 常情况下即为球晶的半径增长速度); $\Delta f$ 为晶体熔 融体自由能; $k$ 为波尔兹曼常数; $T_{c}$ 为结晶温度; $G_{i}$ 为一系数, 如式(43)所示, 其中, $l_{u}$ 为高分子中的单 体段长度; $N_{0}$ 为二次成核首链节的个数; $J$ 为一个 随温度变化的系数; $a_{0}$ 与 $b_{0}$ 分别为单位链节形成晶 体的底面的长宽; $R$ 为理想气体常数; $U^{*}$ 为链段传 输所需自由能; $j$ 为一系数, 取决于二次成核的生长 模式, 随着生长方式的不同, 可有 2 和 4 两种取值.

各个参数的物理意义详见图 5 .

图 5 为 L-H 理论所建立的晶体生长模型, 链节首 先附于晶片底基上, 再层层折叠吸附, $G$ 即为所求的 晶片生长速度, $g$ 为铺展速度. $a_{0}$ 与 $b_{0}$ 分别为单位 链节形成晶体的底面的长宽.

由 2.2 部分所建立的磁场下一次成核速率的理 论, 可知, 聚合物在磁场下的结晶自由能会产生一定 的变化, 我们用 $\Delta f_{m}$ 替代式(42)与(43)中的 $\Delta f$, 得到 下式:

$$
G=G_{i} \exp \left(-U^{*} / R\left(T_{c}-T_{\infty}\right)\right) \exp \left(-\frac{j \sigma \sigma_{e}}{K T_{c} \Delta f_{m}}\right)
$$

$$
\text { 其中 } G_{i}=\frac{N_{0} b_{0} J}{l_{u}}\left[\frac{k T_{c}}{b_{0} \sigma}-\frac{k T}{2 b_{0} \sigma+a_{0} b_{0} \Delta f_{m}}\right]
$$

$$
\Delta f_{m}=\Delta f-T\left(S_{a^{\prime}}-S_{a}\right)-\Delta F_{m}
$$

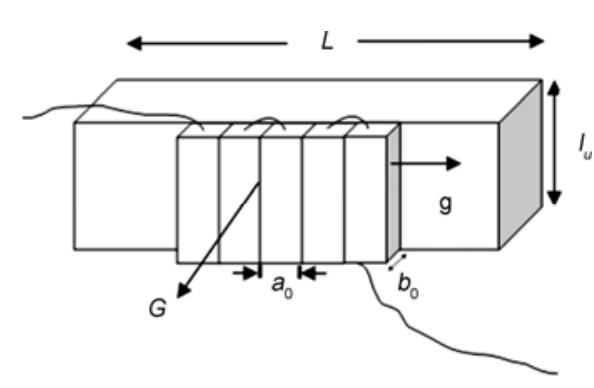

图 5 L-H 理论所建立的晶体生长模型 
由式(44)可知, 在一定过冷度下, 当 $x_{\mathrm{c}}>x_{\mathrm{a}}$ 时, 随着磁感强度 $B$ 增加, $\Delta F_{\mathrm{m}}$ 变小, 晶片生长速率 $G$ 将增大, 晶体生长加速; 当 $x_{\mathrm{c}}<x_{\mathrm{a}}$ 时, 随着磁感强度 $B$ 增加, $\Delta F_{\mathrm{m}}$ 变大, $G$ 则会降低, 晶体生长速率降低. 同时, 如果有“似液晶相”的存在, 也会增加结晶体生 长速率. 与 2.5 部分的论述相似, 这两个效应有可能 互相削弱, 且只有当这些效应的叠加产生的自由能 变化足够大时, 即与结晶体自由能数量级接近时, 才 可以产生可观测的变化.

根据以上对于聚合物在磁场下一次成核与生长 的分析, 可见, 成核率与生长速率都受到了磁场的影 响, 且都有相同的趋势, 即当成核率 $I$ 增大时, 生长 速率 $G$ 也增大; 成核速率 $I$ 减小时, 生长速率 $G$ 也减 小. 但是, 它们的变化程度是有一定差异的, 因而, 会导致最终晶体尺寸和成核密度的变化, 聚合物经 过磁场处理后, 晶体可以变得粗大, 也可以变得细 化, 即出现“磁结晶效应”.

\section{4 磁场对于晶体生长整个过程的结晶动力 学影响}

聚合物晶体生长的结晶动力学可由 Avrami 方程 描述 ${ }^{[23 \sim 26]}$, 方程式如下:

$$
\ln (1-x)=-K t^{n}
$$

其中 $n$ 为 Avrami 指数, 一般与成核机理与生长方式 有关, $K$ 则为结晶速率常数, 其表达式为:

$$
K=I \cdot f(G)
$$

$I$ 为一次成核速率, $f(G)$ 为各种不同生长方式的晶 体生长速率, 是 $G$ 的函数, 以形成球晶为例, 有下 式:

$$
f(G)=\frac{4 \pi}{3} G^{3}
$$

则式(45)可写为:

$$
\ln (1-x)=-(I \cdot f(G)) t^{n}
$$

由上式可见, 整个晶体的总结晶度与时间的关 系, 是由一次成核速率 $I$ 与晶片生长速度 $G$ 决定. 根 据结晶动力学方程(式(48)) 以及磁场下的结晶理论可 知(式(41)(44)), $G$ 与 $I$ 随着各相磁化率的不同而变 化, 且变化趋势一致, 当它们都趋于增加时, 整个体 系的结晶速率 $K$ 可能会明显加快, 这意味着磁场有
可能有效地提高聚合物的结晶速率，反之则减慢.

这一结论与日本学者 Kimura 的研究报道一致. 他在研究 $\mathrm{iPS}^{[6]}$ 体系在强磁场中的结晶动力学时, 发 现其结晶速率约提高了一倍, 见图 6 .

图 6 是 iPS 经过磁场处理的与未经磁场处理后, 根据 FTIR 表征的相对结晶度绘制的结晶动力学曲 线对比图. 图中纵坐标 $I_{983} / I_{1027}$ 代表了两个红外特 征峰之间的比值, 这一比值可代表样品的结晶度大 小. 从图中通过分析聚合物在磁场中的结晶动力学 曲线可以明显发现半结晶期由 $110 \mathrm{~min}$ 左右减少到 $60 \mathrm{~min}$ 左右, 缩短了约一倍, 这说明, 在磁场下的 iPS 结晶速率提高了约一倍, 磁场加速了其整个结 晶过程，且 Kimura 在实验中也发现了前述的“似液 晶相”, 但 Kimura 并未对结晶动力学曲线的改变做 出详尽的分析. 本文建立的理论在一定程度上可以 解释磁场下的此种现象, 即可能是由于磁场的施加 导致两相之间产生了磁化能差及“似液晶相”结构对 体系的熵值影响, 最终导致体系整个结晶速率的增 加.

\section{5 结论}

本文建立了磁场下聚合物成核与生长的理论. 该理论认为, 磁场的施加会导致聚合物相变时由于 两相有着不同的磁化率而产生一个磁化能差, 当 $x_{c}>x_{a}$ 时, 随着磁感强度 $B$ 增加, 晶体成核速率 $I$ 晶 片生长速率 $G$ 都将增大, 加速整个结晶过程; 当 $x_{c}<x_{a}$ 时, 随着磁感强度 $B$ 增加, $I$ 与 $G$ 则都会降

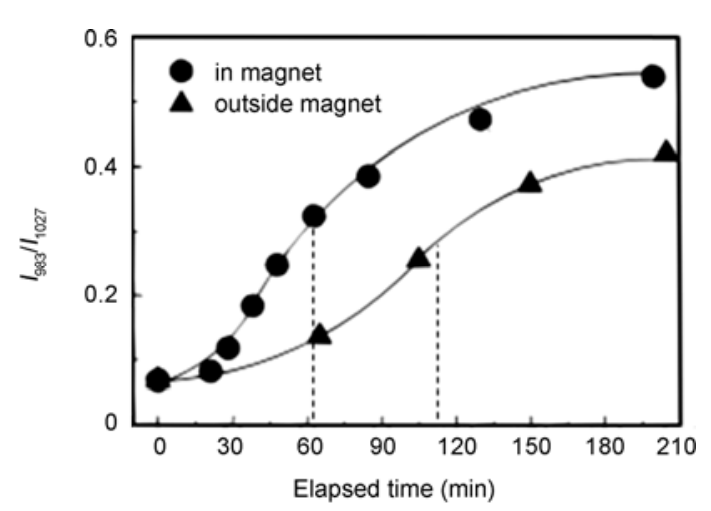

图 6 iPS 经过磁场处理的与未经磁场处理后, 根据 FTIR 表 征的相对结晶度绘制的结晶动力学曲线对比图 ${ }^{[6]}$. 虚线指出 的是半结晶期 
低, 减慢整个结晶过程. 同时, 似液晶相的存在, 也 会增加结晶生长速率, 当这些效应的叠加产生的自 由能变化足够大时, 便可以产生可观测的变化. 这一 理论可以一定程度解释当今学者发现的磁结晶效 应 $^{[12 \sim 14]}$, 且表明, 可以通过施加磁场大面积无接触地 改变聚合物体系的相变自由能, 从而改变其结晶动 力学过程.

并且以 PLLA 为例, 用 Matlab 软件描述了结晶 自由能与各成核临界参数之间的关系, 说明, 在较小 的过冷度下, 成核临界参数可以对结晶自由能的变
化有较大响应, 且可以预见, 可以明显改变聚合物成 核与生长的体自由能的外场等因素, 在一定条件下, 都可能对聚合物的结晶过程产生较明显的影响, 改 变其成核、生长速率进而改变整个结晶动力学方程, 也可能导致晶体形貌的变化, 如晶体的细化等, 它可 能是未来调控晶体结构实现聚集态结构构筑以及调 节成型加工条件的一种环保的手段.

由于这部分的工作处于探索阶段, 需要更多确 切的实验数据来进行验证, 进而丰富与完善该理论, 因而并未涉及更多可能的效应, 如磁滞链传输等.

\section{参考文献}

1 胡爱军, 郑捷. 磁场影响溶液结晶过程研究进展. 江苏化工, 2002, 30: 30-32

2 杨笏, 杨祖荣, 史季芬, 江生南. 磁场对碳酸氢钠结晶动力学的影响. 北京化工大学学报, 1997, 24: 1-2

3 孙佳江, 郭祀远. 磁场处理对蔗糖结晶速度的影响. 齐齐哈尔大学学报, 1995, 02

4 罗漫, 陆柱. 磁场对 $\mathrm{CaCO}_{3}$ 结晶过程的影响. 华东理工大学学报, 2000, 26: 177-179

5 Sata H, Kimura T, Ogawa S, Ito E. Magnetic orientation of poly(ethylene-2,6-naphthalate) during crystallization from melt. Polymer, 1998, 39(25): 6325-6330

6 Ezure H, Kimura T, Ogawa S, Ito E. Magnetic orientation of isotactic polystyrene. Macromolecules, 1997, 30(12): 3600-3605

7 Kawai T, Kimura T. Magnetic orientation of Isotactic polypropylene. Polymer, 2000, 41: 155-159

8 Kimura T, Kawai T, Sakamoto Y. Magnetic orientation of poly(ethylene terephthalate). Polymer, 2000, 41: 809-812

9 Florian E, Thomas TA. Controlling the orientation of semicrystalline polymers by crystallization in magnetic fields. Macromolecules, 2003, 36: $8685-8694$

10 刘强. 磁场诱导下的等规聚丙烯薄膜显微结构分析. 沈阳化工学院学报, 2005, 19(1): 28-31

11 滕蒙雪, 班春燕, 崔建忠. 聚甲醛在强磁场下结晶的形貌分析. 高等学校化学学报, 31(2): 387-390

12 Levin MN, Matveev NN. Effect of pulsed magnetic field treatment on the crystallization and melting of organsilicon polymeric materials. Russian J Phys Chem, 2001, 75(10): 1728-1732

13 Levin MN, Postnikov VV, Matveev NN. Effect of pulsed magnetic field treatment on the crystallization and melting of poly(ethylene oxide). Russian J Phys Chem, 2003,77(4): 675-678

14 Levin MN. Effect of pulsed magnetic fields on crystallization of polymers. Mat Res Soc Symp Proc, 2003, 734: 415-421

15 肖学山, 李维火, 董远达, 莫志深, 王庆, 徐晖. 聚合物熔体在强静电场下成核与长大的理论分析. 上海大学学报, 2001, 7(3): $235-23$

16 Kelton KF. In crystal nucleation in liquids and glasses. Solid State Phys, 1991, 45: 75-177.

17 何曼君. 高分子物理(第三版). 上海:复旦大学出版社, 2007: 156

18 Donald G. A literature review of poly(lactic acid), J Polym Environ, 2002, 9(2): 63-68

19 Wu LB, Hou HB. Isothermal cold crystallization and melting behaviors of poly(L-lactic acid)s prepared by melt polycondensation. $J$ Appl Polym Sci, 2010, 115: 702-708

20 de Gennes PG. Soft matter. Rev Mod Phys, 1992, 64: 645-648

21 Hoffman JD, Davis GT, Lauritzen JI. Treatise on Solid State Chemistry. ed. Hannay NB. New York: Plenum Press, 1976, 3: 497-614

22 Snyder CR, Marand H. Effect of chain transport in the secondary surface nucleation based flux theory and in the Lauritzen-Hoffman crystal growth rate formalism. Macromolecules, 1997, 30: 2759-2766

23 Avrami MJ. Kinetics of phase change. I. General theory. Chem Phys, 1939, 7: 1103-1104

24 Avrami MJ. Kinetics of phase change. II. Transformation-time relations for random distribution of nuclei. J Chem Phys, 1940, 8(2): $212-224$ 
25 Avrami MJ. Kinetics of phase change. III. Granulation, phase change, and microstructure. J Chem Phys, 1941, 9(2): 177-184

26 Hiemenz PC. Polymer Chemistry: The Basic Concepts Marcel Dekker. New York: Marcel Dekker, 1984: 219-220

\title{
A theory of polymer crystallization kinetics influenced by magnetic field
}

LIU Li ${ }^{1,2 *}$, WU WenTan ${ }^{1}$

1 Polymer Department, Shanghai University, Shanghai 201800, China

2 Key Laboratory of Hollow Fiber Material and Membrane, Ministry of Education; Tianjin Poly-technic University, Tianjin 300387, China

*Corresponding author (email: liuli@staff.shu.edu.cn)

\begin{abstract}
The mechanism of the magnetic field influence on polymer bulk crystallization has been discussed, which included nucleation and crystal growth. We have constructed relative equations of crystallization kinetics based on the classic thermodynamic theory. The equations show that the "magnetocrystallization effect" (MCE) caused by the difference of magnetic energy between crystal and amorphous phases. It came from the difference of magnetic susceptibilities between crystal and amorphous phases. The "like-liquid-crystal phase" induced by magnetic field in some polymer is another possible reason of MCE, which have low entropy and change the free energy in crystallization. They could affect the process of nucleation, crystal growth, the entire formula of crystal kinetics. We used "Matlab" software to figure the function of nucleation critical parameters and crystal free energy, the pictures indicated that small free energy changed by the temperature or magnetic field could greatly impact on the values of nucleation critical parameter with low degree of supercooling.
\end{abstract}

Keywords: magnetic field, polymer bulk crystallization, thermodynamics, Matlab software 